\title{
Structure and vibrational properties of carbon tubules
}

\author{
N CHANDRABHAS ${ }^{1}$, A K SOOD ${ }^{1,4}$, D SUNDARARAMAN ${ }^{2}$, S RAJU $^{2}$, \\ V S RAGHUNATHAN ${ }^{2}$, GVN RAO ${ }^{3}$, V S SASTRY ${ }^{3}$, T S RADHAKRISHNAN ${ }^{3}$, \\ Y HARIHARAN $^{3}$, A BHARATHI $^{3}$ and C S SUNDAR ${ }^{3}$ \\ ${ }^{1}$ Department of Physics, Indian Institute of Science, Bangalore 560012, India \\ ${ }^{2}$ Metallurgy Division, ${ }^{3}$ Materials Science Division, Indira Gandhi Centre for Atomic Research, \\ Kalpakkam 603 102, India \\ ${ }^{4}$ Also at Jawaharlal Nehru Centre for Advanced Scientific Research, IISc Campus, Bangalore \\ 560012, India
}

MS received 11 February 1994; revised 6 April 1994

\begin{abstract}
The structure of multilayered carbon tubules has been investigated by electron microscopy and X-ray diffraction. The structure of tubules is characterized by disorder in the stacking of cylindrical graphene sheets. Raman scattering measurements have been carried out in tubules and compared with graphite. The observed features in the Raman spectra in tubules can be understood in terms of the influence of disorder. The additional Raman modes predicted for single layer carbon tubules have not been observed.
\end{abstract}

Keywords. Nanoparticles; tubule; graphite; Raman spectroscopy.

\section{PACS Nos 78.30; 61·46}

\section{Introduction}

Since the first observation of carbon tubules by Iijima [1] there has been a tremendous excitement with respect to their novel structure, and physical properties and their potential for applications in nanotechnology [2]. The tubular morphology usually consists of several concentric folded graphitic sheets, capped at ends. Theoretical calculations $[3,4]$ of single tubules have predicted exotic electronic properties ranging from metallic to semiconductor behaviour depending on the chirality and diameter of the tubule. The vibrational properties have been extensively investigated theoretically by Dresselhaus and co-workers [5, 6] with specific reference to Raman and infra-red active modes. The number of Raman and infrared active modes are only dependent on the symmetry, but the phonon frequencies are seen to depend on the tubule diameter. While most of the theoretical interest has been on a single tubule, and recently some experimental methods for the preparation of predominantly single tubules have been obtained [7], the experiments so far have been only in multi-layered tubules.

In the present work, studies on the structure and vibrational properties of multilayered tubules are reported. The structural studies have been carried out using electron microscopy and X-ray diffraction. The vibrational properties have been investigated using Raman spectroscopy. During the course of this study, we became aware of the work of Hiura et al [8] on these systems. These results will be compared with our present study. In addition, new results especially in low-frequency range of Raman scattering will be presented. 


\section{$N$ Chandrabhas et al}

\section{Experimental details}

Following the prescription of Ebessen and Ajayan [9] for the large scale synthesis of carbon nanotubes, arcing between graphite electrodes (5N5 pure from Johnson and Mathey, USA) was carried out under 500 torr He atmosphere using a KratscmerHuffman type apparatus described in detail elsewhere [10]. When the arcing was carried out at $20 \mathrm{~V} \mathrm{AC}, 125 \mathrm{~A}$, the smaller graphite electrode of $6 \mathrm{~mm}$ diameter was consumed and a carbonaceous deposit gets formed on the larger $10 \mathrm{~mm}$ diameter graphite electrode. The central core of the deposit, rich in tubules [9] was scooped out, crushed, suspended in ethanol, and ultrasonically agitated for about an hour and then allowed to settle down. A drop or two of the supernatant was transferred to holey carbon film for electron microscopy observations, which were carried out using Philips EM 400 T and JEOL EXII microscopes. The extracted powder was used for X-ray and Raman scattering experiments. X-ray diffraction measurements were carried out with $\mathrm{Cu}-\mathrm{K}_{\alpha}$ radiation, using Siemens D-500 diffractometer, operating in the 9-29 Bragg-Brentano arrangement. Raman measurements were done in the back scattering geometry using $5145 \mathrm{~A}$ line of argon-ion laser (laser power $\sim 30 \mathrm{~mW}$ ) and DILOR-XY spectrometer equipped with liquid nitrogen cooled CCD detector. The low frequency (less than $200 \mathrm{~cm}^{-1}$ ) spectra were recorded from the sample kept in vacuum to avoid interference with the Raman lines of nitrogen gas. In addition, experiments were also done on the powder obtained from the outer shell of the carbonaceous deposit and on the starting graphite sample.

\section{Results and discussion}

\subsection{Electron microscope studies}

Electron microscope observations indicated a preponderance of multi layered carbon tubules along with occasional presence of polyhedral particles having spherical shell structure. Figure 1(a) shows a typical transmission electron micrograph of a nested carbon tubule in which the diameter varies from $15 \mathrm{~nm}$ to $50 \mathrm{~nm}$. As has been discussed earlier [11] such a change in diameter is associated with the presence of pentagons and heptagons in the hexagonal graphitic sheet. Figure 1(b) shows the selected area diffraction pattern taken with the electron beam perpendicular to the tubule axis. As discussed by Iijima [9], the sharp (001) spots arise from the parallel graphitic sheets and point to the co-axial arrangement of graphitic sheets in the tubule. The ring-like spots which are a superposition of the $(h k 0)$ reflections (the (100) and (110) can be seen in figure 1(b)) arise because the successive hexagonal sheets are not under exact superposition.

The high resolution microscope image of the (002) lattice fringes is shown in figure 1 (c). The tubule pictured in figure 1 (c) shows one half of the tubule containing about 46 layers and has a core of about $2 \mathrm{~nm}$. One of the important features seen in this figure is the presence of localized region with significant faulting in the stacking of graphene sheets. It is well-known [9] that for tubular arrangement of graphitic sheets, the $\mathrm{ABABAB}$... stacking present in ideal graphite structure is not possible and a turbostatic arrangement is expected. The present high resolution picture indicates the presence of stacking faults which are shown in detail in figure 1(d), which also shows in some regions considerable puckering from true planarity. A schematic illustrating the possible stacking of graphene layers from $\mathrm{ABAB}$... type to $\mathrm{ABCABC}$... 


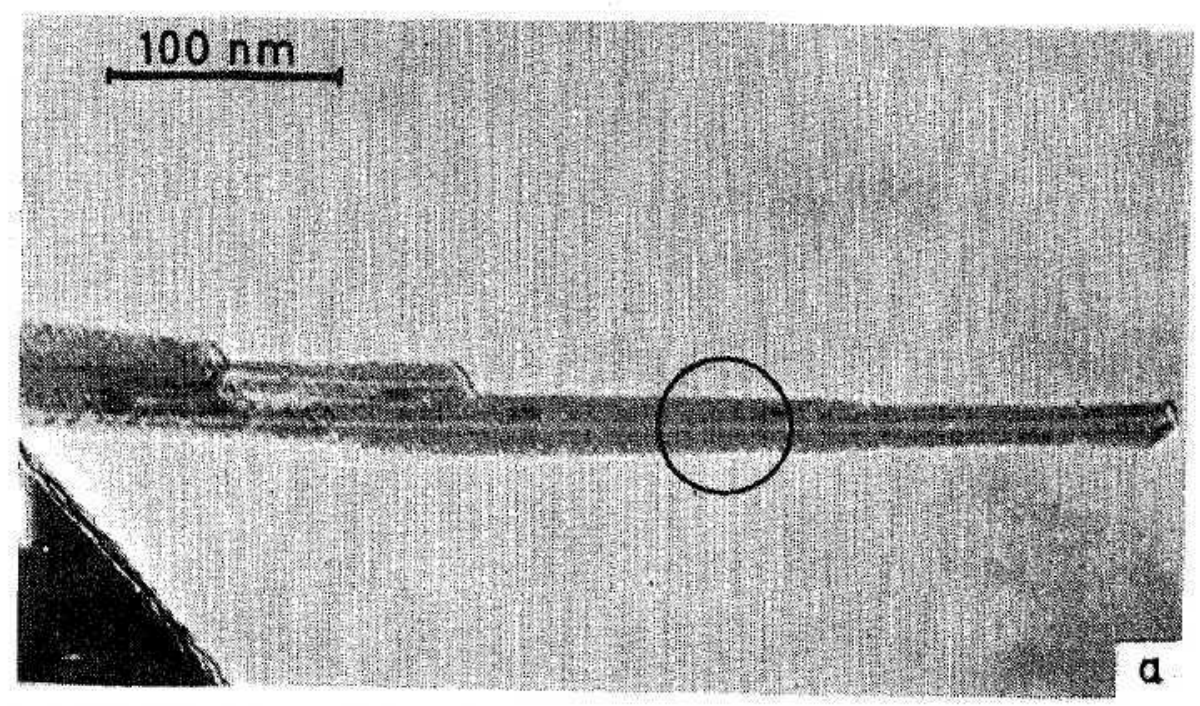

Figure 1(a). Transmission electron micrograph of multi-layered carbon tubule showing the nested arrangement. The diameter in the ringed region of tubule is $25 \mathrm{~nm}$ and in the thinner region is $15 \mathrm{~nm}$.

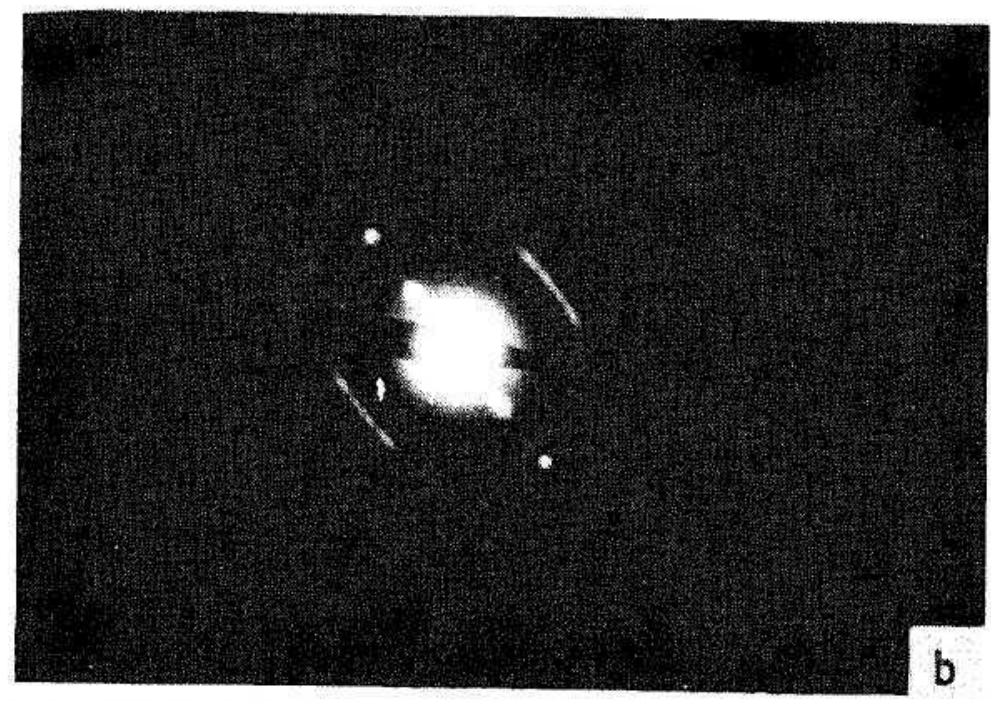

Figure 1(b). Selected area diffraction from the circled portion of tubule.

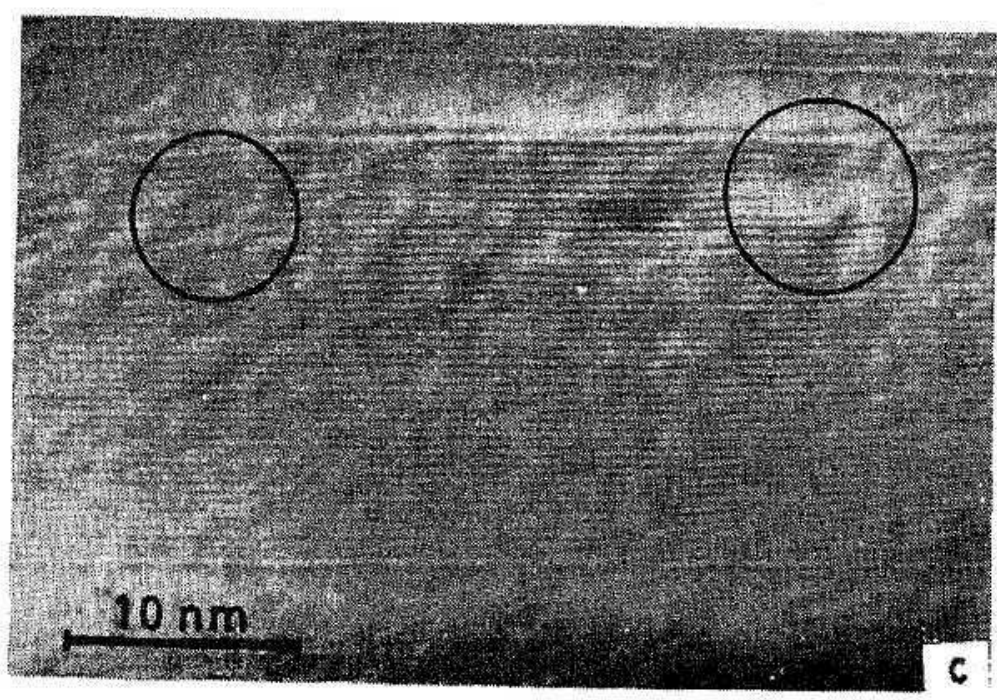

Figure 1(c). High resolution electron micrograph of tubule. Note the significant local deviations of (002) fringes from planarity. Dislocation type defects are also observable. 


\section{$N$ Chandrabhas et al}

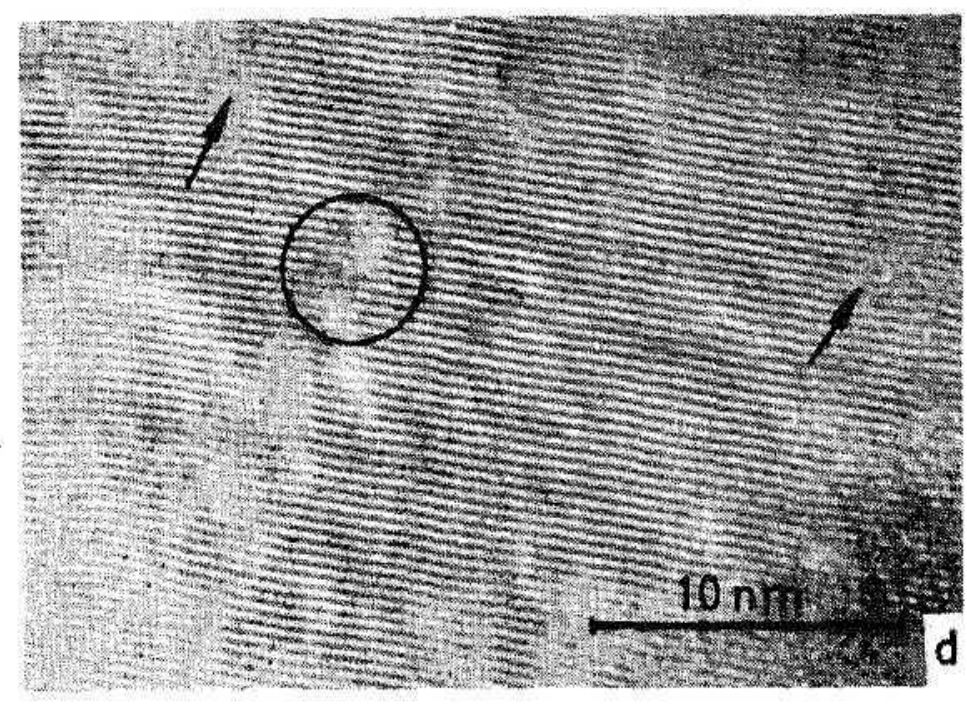

Figure 1 (d). A region similar to that shown in (c), but is slightly enlarged showing clearly the puckering of planes and dislocation type defects.

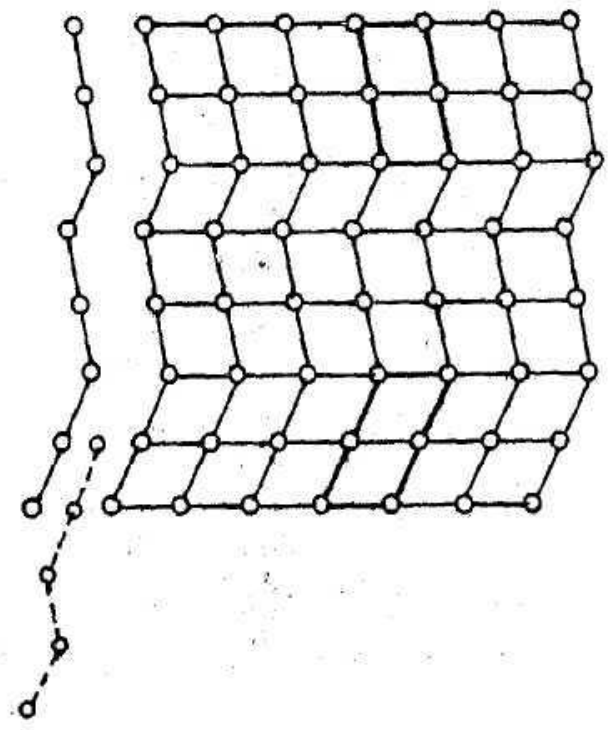

Figure 2. Schematic illustrating the stacking of graphene layers giving rise to defects as shown in figures 1(c) and (d). The illustrated sequence corresponds to a fcc stacking with a twin boundary in between. A dislocation like arrangement is also illustrated through dotted lines. A unit cell like configuration is outlined by strong lines. 


\section{Structure and vibrational properties of carbon tubules}

(rhombohedral type) is shown in figure 2. The restoration of a particular stacking sequence could be interpreted in a multitude of ways by allowing for planar defects: this stacking fault defects are mandatory for larger diameter tubes and may even serve as potent nucleating site for another tubular variant. Further, in view of the considerable influence that these defects may have on the electronic and vibrational properties, detailed studies of defects in multi layered tubules are called for.

\subsection{X-ray diffraction}

The X-ray diffraction pattern of the starting graphite powder and that of the tubule powder are shown in figures 3 (a) and $3(\mathrm{~b})$, respectively. The diffraction pattern of graphite can be indexed to a hexagonal cell with lattice parameters $a-2.46 \mathrm{~A}$ and $c=6.72 \mathrm{~A}$ in accordance with the results reported in literature [12]. In the diffraction pattern of the tubule, the (002) and (004) lines can be clearly seen to be shifted to lower angles indicating an increase in the ' $c$ ' lattice parameter to 6.85 A. The reflections indexed as (10) and (11) (i.e., diffraction from a two dimensional lattice) are broad and asymmetric and are similar to that seen in turbostatic graphite [13]. From the positions of the (10) and (11) reflections, the ' $a$ ' lattice parameter can be evaluated to be $2.46 \mathrm{~A}$. Thus in the tubule structure, while the intra-layer distance between the carbon atoms is unaffected that between the layers is larger than in graphite [14]. The diffraction pattern from the outer shell is shown in figure 3(c). It is interesting to note that the diffraction pattern is similar to that of tubules (figure 3(b)) though no tubules have been observed by electron microscopy. It is also worth pointing out that the diffraction pattern of the outer shell does not correspond to that of amorphous carbon.

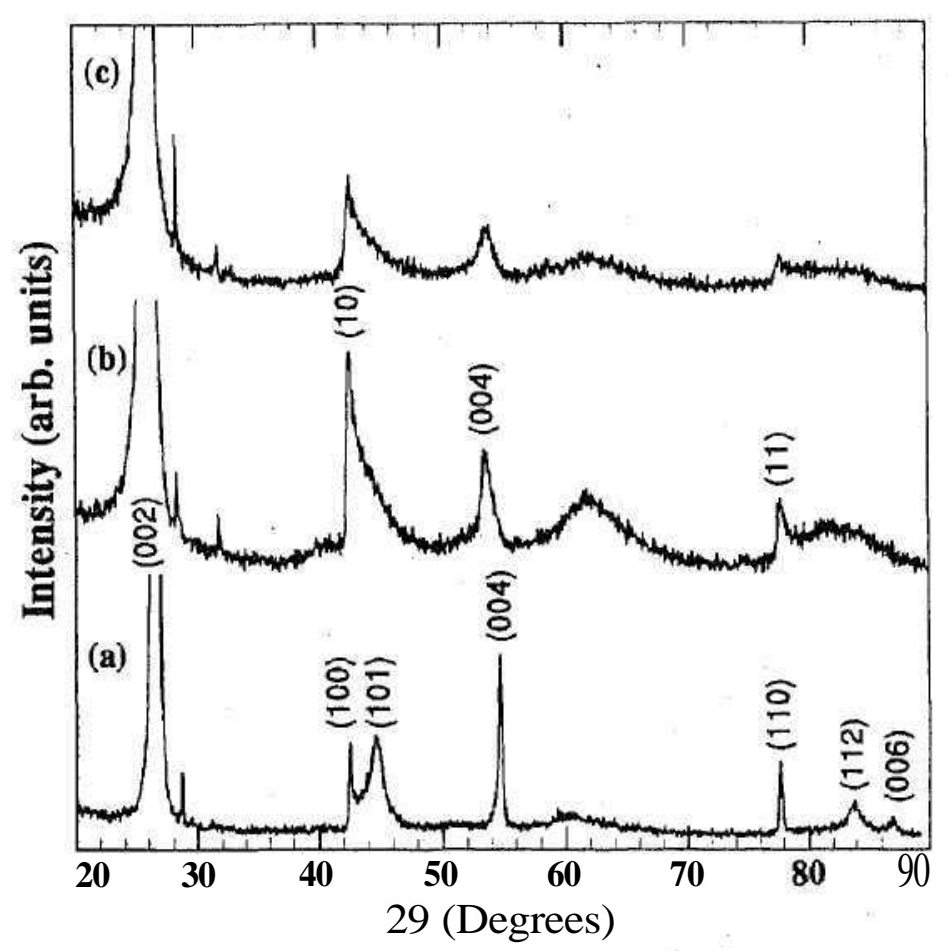

Figure 3. X-ray diffraction pattern in (a) graphite, (b) tubules from the inner core and (c) outer shell of the carbonaceous deposit. 


\section{$N$ Chandrabhas et al}

\subsection{Raman spectroscopy}

Raman spectroscopy has proven to be a powerful technique for the study of graphite, its intercalation compounds [15] and fullerenes [16]. Apart from providing information on the zone centre Raman active phonons, the Raman spectra (RS), in the case of disordered solids, contains information on the phonon density of states (PDOS). Further, the degree of $s p^{2}-s p^{3}$ bonding also leaves characteristic Raman f i $\mathrm{n} g$ e $r$ prints.

Pristine graphite crystallises according to the $D_{6 h}^{4}$ and has nine vibrational optic modes: $2 E_{2 g}(R)+2 B_{1 g}+A_{2 u}(I R)+E_{u}(I R)$, where $R$ and $I R$ denote Raman active and infra-red active modes respectively. The two Raman active modes are (a) $E_{2 g}^{2}$ at $\omega=1582 \mathrm{~cm}^{-1}$ which involves out pf phase intra-layer displacements and (b) $E_{2 g}^{1}$ at $\omega=42 \mathrm{~cm}^{-1}$ which involves in phase inter-layer displacements resulting in rigid shearing motion between graphite layers [15]. Jishi et al [6] have calculated the allowed Raman modes of a tubule using a zone-folding technique for two-dimensional dispersion curves from a graphene layer, which indicate that there are 15 allowed Raman modes for each diameter tube: Whereas the frequency of the allowed modes depend on the tube diameter and the chiral angle, the number of modes is independent

\section{'diame'}

- Figure 4 shows the RS in the region of the $E_{2 g}^{2}$ mode $\left(1250-1750 \mathrm{~cm}^{-1}\right)$ of (a) high purity graphite (b) inner core of the carbonaceous deposit containing tubules and (c) the outer shell of the deposit. The PDOS calculated in terms of an axially symmetric Born-von Karman force-constant model [17] is shown in figure 4(d) which shows peaks at about 1335,1425 and $1610 \mathrm{~cm}^{-1}$. In the case of graphite, the sharp Raman

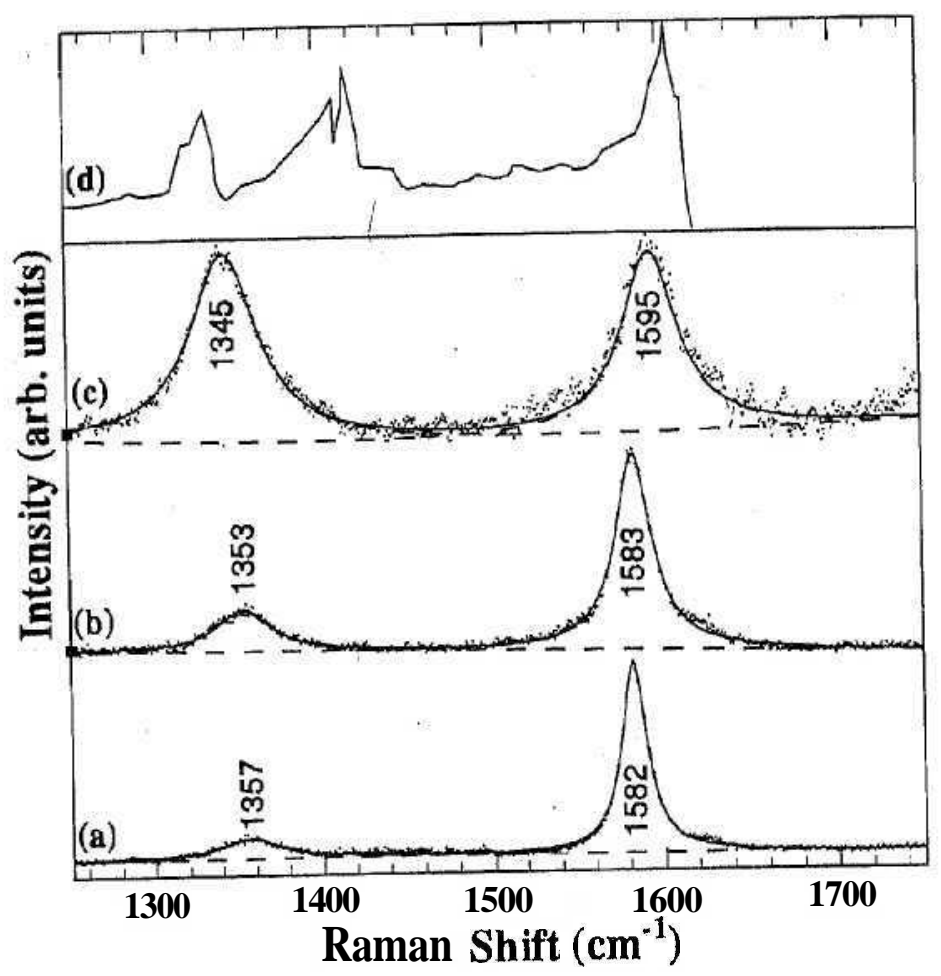

Figure 4. Raman spectra in the range $1250 \mathrm{cmo}^{1} 1750 \mathrm{~cm}$ in (a) graphite, (b) tubules in the inner core and (c) the outer shell of the carbonaceous deposit. The calculated PDOS (Ref. 17) is shown in (d). 


\section{Structure and vibrational properties of carbon -tubules}

line at $1582 \mathrm{~cm}^{-1}$ is seen. The broad hump centered at $1357 \mathrm{~cm}^{-1}$ is related to the presence of disorder/finite-size effects leading to break down in the wave vector selection rules and a peak in the RS occurs near the maxima in the PDOS [18]. The corresponding Raman line of the inner core (figure $4(\mathrm{~b})$ ) is at $1353 \mathrm{~cm}^{-1}$. The peak positions are (i) 1582 and $1357 \mathrm{~cm}^{-1}$ for graphite (figure 4(a)) (ii) 1583 and $1353 \mathrm{~cm}^{-1}$ for the inner core (figure 4(b)) and (iii) 1595 and $1345 \mathrm{~cm}^{-1}$ for the outer deposit (figure $4(\mathrm{c})$ ). As we go progressively from graphite to the inner core and the outer shell (figure 4(a) to figure 4(c)), the following features in the RS are observed: (1) The Raman line associated with the $E_{2 g}^{2}$ mode broadens and shifts to higher wave number, (2) The $1357 \mathrm{~cm}^{-1}$ line associated with the disorder increases in relative intensity and shifts towards lower wave number.

As already remarked the intensity of Raman lines in a disordered material reflects the dominant features in the PDOS $[15,18]$. Theoretical calculations of the PDOS of graphite by Leung et al [19] using the phonon dispersion relations calculated by Maeda et al [20] and by Niclow et al [17] indicate that the largest peak in the PDOS occurs at $\sim 1610 \mathrm{~cm}^{-1}$, upshifted from the zone-centre Raman active phonon at $1582 \mathrm{~cm}^{-1}$. In the case of tubules in the inner core and the outer shell, having considerable disorder, this feature in the PDOS is probed, resulting in a up-shift of the Raman line. This can be clearly seen as the small shoulder at $1620 \mathrm{~cm}^{-1}$ seen even in graphite (figure 4(a)) merges with the $1582 \mathrm{~cm}^{-1}$ line leading to a broad peak at $1595 \mathrm{~cm}^{-1}$ in the outer deposit (figure 4(c)). The next dominant feature in the PDOS is [17-20] at $1355 \mathrm{~cm}^{-1}$ which accounts for the small peak in graphite shown figure 4(a). As we go from figure 4(a) to figure 4(c), this peak is seen to increase in

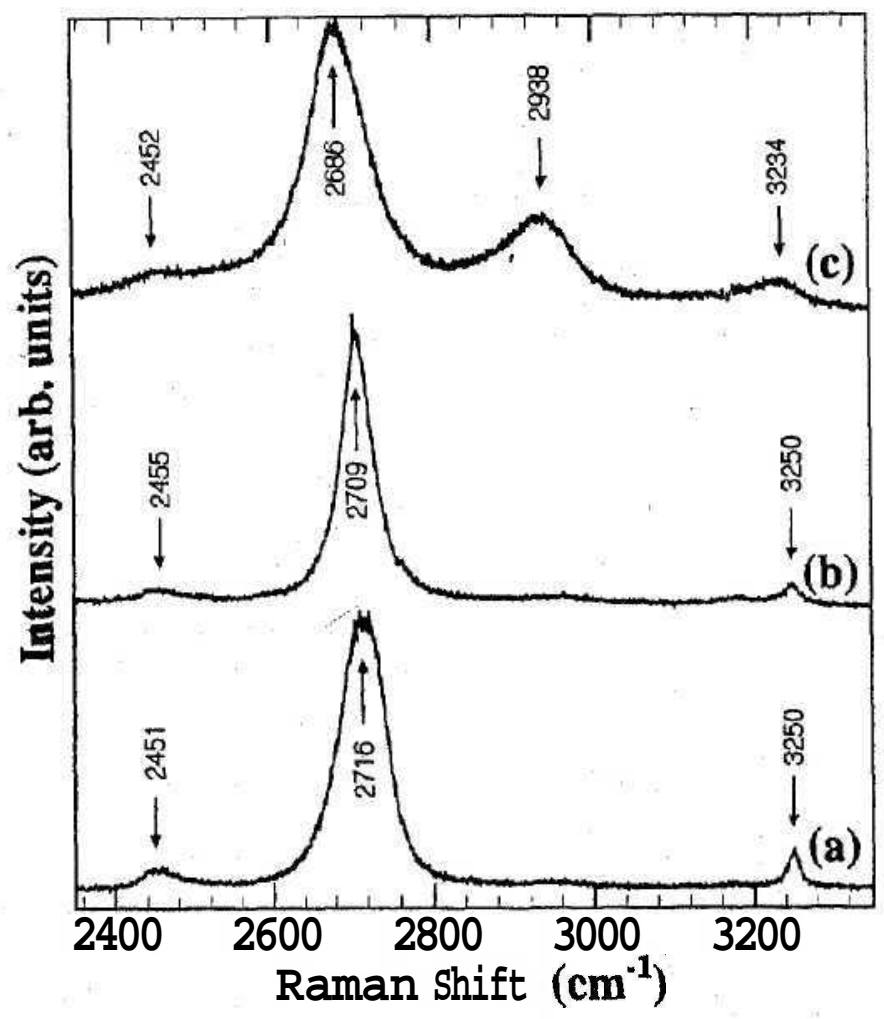

Figure 5. Second-order RS in the range $2400 \mathrm{~cm}^{-1}$ to $3200 \mathrm{~cm}^{-1}$ in three samples as in figure 4. 


\section{$N$ Chandrabhas et al}

relative intensity and shift to lower wave number. This softening of phonons can be related to the larger c-axis lattice parameter in tubule and outer shell as compared to graphite (cf. §3.2). Thus it is seen that the progressive changes of both the line positions and intensity of the Raman lines seen in figure 4 can be qualitatively understood in terms of the increase in disorder as we go from figure 4(a) tofigure 4(c).

The second-order RS are associated with the phonons of wave vector $\mathrm{q}$ and $-\mathrm{q}$ and hence are sensitive to PDOS $[15,18]$. Figure 5 shows the second-order RS in the range $2400 \mathrm{~cm}^{-1}$ to $3200 \mathrm{~cm}^{-1}$. The progressive downshift of the $2716 \mathrm{~cm}^{-1}$ line (overtone corresponding $1357 \mathrm{~cm}^{-1}$ mode) can be seen from figure 5 (a) to figure 5(c) and this is consistent with the downshift seen in the first-order spectra (cf. figure 4). The other two lines at $2451 \mathrm{~cm}^{-1}$ and $3250 \mathrm{~cm}^{-1}$ can be accounted in terms of combination of the peak at $1620 \mathrm{~cm}^{-1}$ and $860 \mathrm{~cm}^{-1}$ phonon and of the two $1620 \mathrm{~cm}^{-1}$ modes. The line at $2938 \mathrm{~cm}^{-1}$ seen only in the case of outer shell (figure 5(c)) can be a combination of the phonons of frequency $1595 \mathrm{~cm}^{-1}$ and $1345 \mathrm{~cm}^{-1}$ peaks. This band also occurs in the RS of the glassy carbon reported by Hiura et al [8]. This is interesting because even though the outer deposit is not X-ray amorphous (figure 3(c)), the RS resembles that of the glassy carbon.

The results of Raman experiments in the range of $200 \mathrm{~cm}^{-1}$ to $1250 \mathrm{~cm}^{-1}$ were characterized by poor signal to noise $(\mathrm{S} / \mathrm{N})$ ratio. In order to improve the $\mathrm{S} / \mathrm{N}$ ratio, these measured spectra were subjected to discrete wavelet transform filtering [21] and the results are shown in figure 6 for graphite (curve (a)), inner deposit (curve (b)) and the outer deposit (curve (c)). The features in the RS appear due to relaxation of Raman selection rules due to disorder and hence should map the PDOS which is shown from the known calculations [17] in figure 6(d). The peaks in PDOS occur at 468 and $\sim 770 \mathrm{~cm}^{-1}$. Following Nemanich and Solin [18], the $\sim 770 \mathrm{~cm}^{-1}$ peak is associated with the M-point zone boundary phonon modes with 'out-of-plane' and 'in-plane' displacements whereas the $468 \mathrm{~cm}^{-1}$ mode arises solely from the M-point mode with 'out-of-plane' displacement. The RS in figure $6(\mathrm{a}-\mathrm{c})$ do show bands near $470 \mathrm{~cm}^{-1}$. The bands near $700 \mathrm{~cm}^{-1}$ in the tubule and outer deposit perhaps correspond to the $1770 \mathrm{~cm}^{-1}$ band in the PDOS. The downward shift of the band can arise due to larger c-axis lattice parameter in comparison to graphite, as also reflected in figure 4. The spectrum in figure 6(c) also shows peaks at $\sim 860$ and $1120 \mathrm{~cm}^{-1}$ which are not prominent in the PDOS. The $860 \mathrm{~cm}^{-1}$ band is seen to contribute to the combination mode at $2451 \mathrm{~cm}^{-1}$ (figure 5). The additional Raman modes predicted in this spectral range by Jishi et al [6] for single tubules have not been observed.

Figure 7 shows the RS of tubules in the low frequency range of 30 to $150 \mathrm{~cm}^{-1}$. The $E_{2 g}^{\dot{1}}$ mode of graphite corresponding to the intra layer shear mode has been identified at $42 \mathrm{~cm}^{-1}$ and $45 \mathrm{~cm}^{-1}$ based on Raman scattering [22] and neutron diffraction [17] experiments. In the case of tubules Raman peak at $49 \mathrm{~cm}^{-1}$ can be seen clearly in addition to the lines at 66 and $77 \mathrm{~cm}^{-1}$ which are the laser plasma lines. It is a moot question whether the Raman line in the tubule is slightly upshifted from the intra-layer shear mode in graphite. First we note that in going from graphite to tubules, the intra-layer carbon distance is not affected (cf. § 3.2). In addition, the three carbon-carbon bond angles are also not very different [23] from 120 degrees due to the cylindrical curvature of graphene sheets (unlike in the case of $\mathrm{C}_{60}$ ). Further, as to whether the small upshift can be related to the features in the PDOS (cf. figure 4), it may be pointed out that theoretical calculations $[17,19]$ do not indicate any peaks in the PDOS in this spectral range. Thus the Raman line at $49 \mathrm{~cm}^{-1}$ can be taken to be similar to that in graphite with no special significance with respect to either 
Structure and vibrational properties of carbon tubules

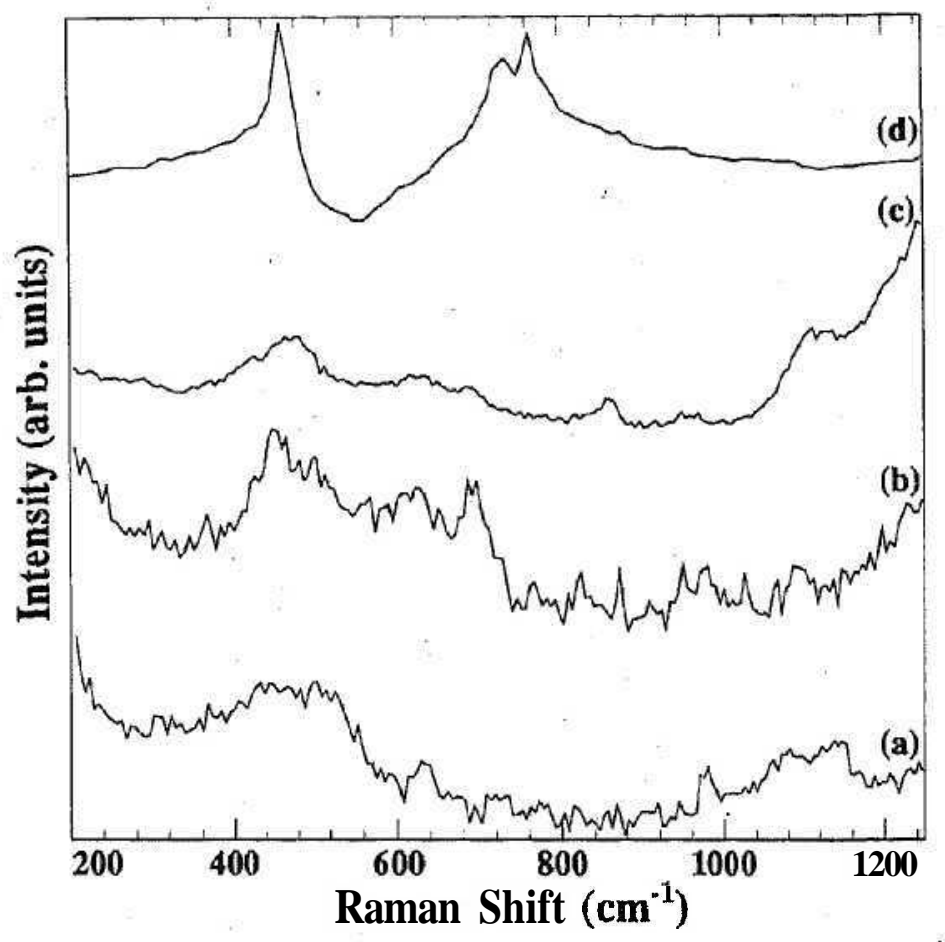

Figure 6. Raman spectra in the range $200 \mathrm{~cm}^{1}$ to $1250 \mathrm{~cm}^{1}$ in (a) graphite, (b) tubules and (c) outer shell. These spectra have been subjected to discrete wavelet transform filtering. The calculated PDOS (ref. 17) is shown in (d).

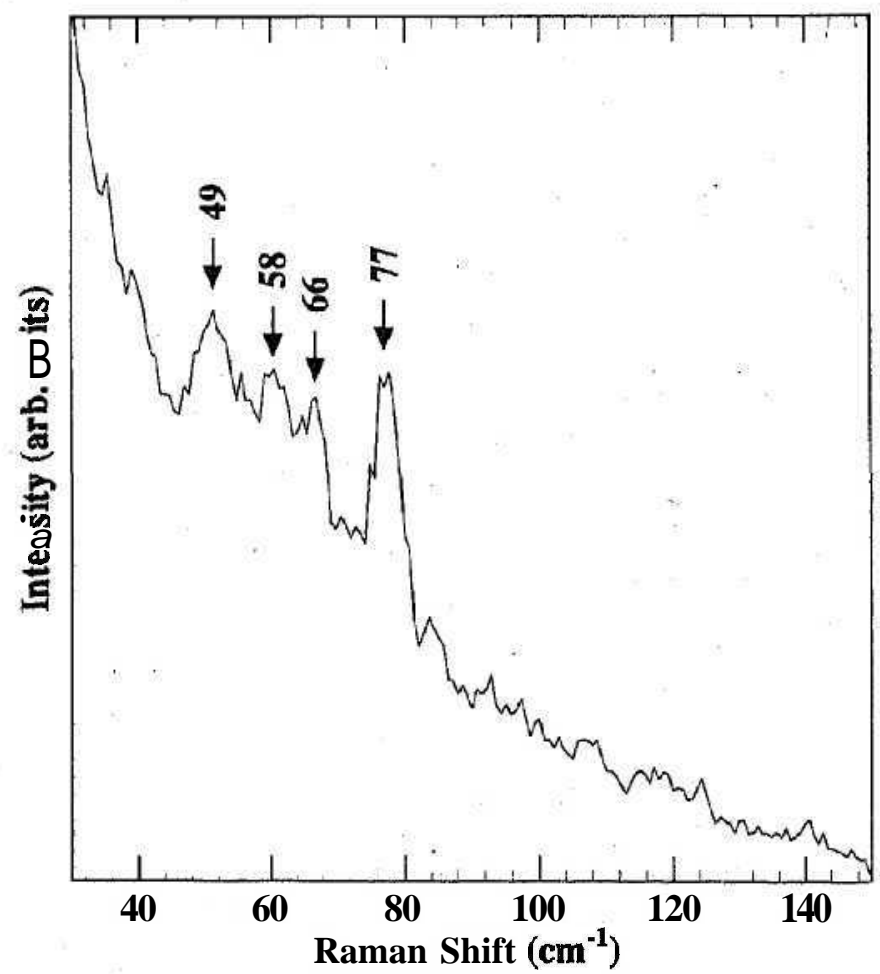

Figure 7. RS of the inner core of the depositin the range $30 \mathrm{~cm}^{1}$ to $150 \mathrm{~cm}{ }^{1}$. 


\section{$N$ Chandrabhas et al}

the tubular structure or disorder in graphitic structure. In figure 7, a small feature at $58 \mathrm{~cm}^{-1}$ can be discerned. It is tempting to associate this with defects in the graphene layer, such as the pentagons and heptagons [11] which will lead to altering of bond distances and angles and thus hardening of tangential modes.

\section{Summary}

The structural studies on multi-layered tubules point to the existence of considerable disorder in the stacking of graphene sheets. The influence of disorder is reflected in the changes in RS with respect to graphite. Additional Raman lines characteristic of single-layered tubules [6] have not been observed in the present experiments. With the recent methods for the synthesis of predominantly single-layered tubules [7], Raman experiments on these may become feasible. In addition to studies on the Raman active tangential modes, infra-red studies of the radial modes will be also of interest.

\section{Acknowledgements}

The authors would like to thank Shri M C Valsakumar for graciously carrying out the discrete wavelet transform filtering of the spectra shown in figure 6. One of the authors (AKS) thanks the Department of Science and Technology for financial assistance.

\section{I}

\section{References}

[1] S Iijima, Nature (London), 358, 220 (1992)

[2] See Materials Science and Engineering B19, (1993)

[3] R Saito, M Fujita, G Dresselhaus and M S Dresselhaus, Appl. Phys. Lett. 60, 2204 (1992)

, [4] N Hamada, S Sawada and A Oshiyama, Phys. Rev. Lett. 68, 631 (1992)

[5] M S Dresselhaus, G Dresselhaus and R Saito, Phys. Rev. B45, 6234 (1992)

[6] R A Jishi, L Venkataraman, M S Dresselhaus and G Dresselhaus, Chem. Phys. Lett. 209, 77 (1993)

R A Jishi, M S Dresselhaus and G Dresselhaus, Phys. Rev. B47, 16671 (1993)

[7] S Iijima, Proc. of MRS meeting (Boston, Dec., 1993);

S C Tsang, P J F Harris and M L H Green, Nature 362, 520 (1993)

[8] H Hiura, T W Ebbesen, K Tanigaki and H Takahashi, Chem. Phys. Lett. 202, 509 (1993)

[9] T W Ebessen and P M Ajyan, Nature (London), 358, 220 (1992)

[10] Y Hariharan, A Bharathi, C S Sundar, V S Sastry, M Yousuf, T S Radhakrishnan, G VN Rao, T Geetha Kumary, N Subramanian, P Ch Sahu, V S Raghunathan and M C Valsakumar, Curr. Sci. 63, 25 (1992)

[11] S Iijama, T Ichihashi and Y Ando, Nature (London), 356, 776 (1992) B I Dunlap, Phys. Rev. B46, 1933 (1992),

[12] P Trucano and R Chen, Nature (London), 258, 136 (1975)

[13] B T Kelley, Physics of graphite (Applied Science Publishers, London, 1981)

[14] Y Saito, T Yoshikawa, S Bandow, M Tomita and T Hayashi, Phys. Rev. B48, 1907 (1993)

[15] M S Dresselhaus and G Dresselhaus, in Light scattering in solids HI, Recent results, edited by M Cardona and G Guntherodt (Springer Verlag, Berlin, 1983), p3

[16] P C Eklund, P Zhou, K A Wang, G Dresselhaus and M S Dresselhaus, J. Phys. Chem. Solids 53, 1391 (1992)

N Chandrabhas, K Jayaram, D V S Muthu, A K Sood, R Seshadri and C N R Rao, Phys. Rev. B47, 10963 (1993)

[17] R Niclow, N Wakabayashi and H G Smith, Phys. Rev. B5, 4951 (1972) 
Structure and vibrational properties of carbon tubules

[18] R J Nemanich and S A Solin, Phys. Rev. B20, 392 (1979)

[19] S Y Leung, M S Dresselhaus and G Dresselhaus, Physica B105, 375 (1981)

[20] M Maeda, Y Kuramoto and C Horie, J. Phys. Soc. Jpn. 47, 337 (1979)

[21] I Daubachies, Wavelets (SIAM, Philadelphia, 1992)

[22] R J Nemanich, G Lucovsky and S A Solin, in Proc. Int. Conf. on Lattice Dynamics, edited by M Balkanski (Flammarion Press, Paris, 1977) p. 619

[23] R A Jishi and M S Dresselhaus, Phys. Rev. B45, 11305 (1992) 